\title{
Validation List no. 95 Validation of publication of new names and new
combinations previously effectively published
outside the IJSEM
}

Correspondence

Jean Euzéby

j.euzeby@envt.fr
The purpose of this announcement is to effect the valid publication of the following new names and new combinations under the procedure described in the Bacteriological Code (1990 Revision). Authors and other individuals wishing to have new names and/or combinations included in future lists should send three copies of the pertinent reprint or photocopies thereof to the IJSEM Editorial Office for confirmation that all of the other requirements for valid publication have been met. It is also a requirement of IJSEM and the ICSP that authors of new species, new subspecies and new combinations provide evidence that types are deposited in two recognized culture collections in two different countries (i.e. documents certifying deposition and availability of type strains). It should be noted that the date of valid publication of these new names and combinations is the date of publication of this list, not the date of the original publication of the names and combinations. The authors of the new names and combinations are as given below, and these authors' names will be included in the author index of the present issue and in the volume author index. Inclusion of a name on these lists validates the publication of the name and thereby makes it available in bacteriological nomenclature. The inclusion of a name on this list is not to be construed as taxonomic acceptance of the taxon to which the name is applied. Indeed, some of these names may, in time, be shown to be synonyms, or the organisms may be transferred to another genus, thus necessitating the creation of a new combination.

\begin{tabular}{|c|c|c|c|c|}
\hline Name/author(s) & Proposed as: & Nomenclatural type ${ }^{\star}$ & Priority $\dagger$ & Reference \\
\hline Aquicella lusitana Santos et al. 2004 & sp. nov. & Strain SGT-39 $(=$ CIP $107650=$ LMG 21647) & 3 & 5 \\
\hline Bryantella Wolin et al. 2004 & gen. nov. & Bryantella formatexigens Wolin et al. 2004 & 1 & 7 \\
\hline Bryantella formatexigens Wolin et al. 2004 & sp. nov. & Strain I-52 $(=$ DSM $14469=$ CCUG 46960$)$ & 1 & 7 \\
\hline Corynebacterium nigricans Shukla et al. 2004 & sp. nov. & Strain CN-1 (=ATCC $700975 \ddagger=$ CIP $107346 \$)$ & 6 & 6 \\
\hline Microtetraspora malaysiensis Nakajima et al. 2004 & sp. nov. & Strain H47-7 (= JCM $11278=$ DSM 44579) & 4 & 4 \\
\hline Promicromonospora pachnodae Cazemier et al. 2004 & sp. nov. & Strain VPCX2 $(=$ DSM $12657=$ NCCB 100020$)$ & 7 & 1 \\
\hline Thermodesulfobiaceae Mori et al. 2004 & fam. nov. & Thermodesulfobium Mori et al. 2004 & 5 & 3 \\
\hline Thermodesulfobium Mori et al. 2004 & gen. nov. & Thermodesulfobium narugense Mori et al. 2004 & 5 & 3 \\
\hline Thermodesulfobium narugense Mori et al. 2004 & sp. nov. & $\begin{array}{l}\text { Strain Na82 (=DSM } 14796=\text { JCM } 11510= \\
\text { NBRC } 100082 \S)\end{array}$ & 5 & 3 \\
\hline
\end{tabular}

For references to Validation Lists 1-71, see Int J Syst Bacteriol 49 (1999) 1325. Lists 72-94 were published in Int J Syst Evol Microbiol 50 (2000) 3, 423, 949, 1415, 1699, 1953; 51 (2001) 1, 263, 793, 1229, 1619, 1945; $52(2002)$ 3, 685, 1075, 1437, 1915; and 53 (2003) 1, 373, 627, 935, 1219, 1701 .

*Abbreviations: ATCC, American Type Culture Collection, Manassas, VA, USA; CCM, Czech Collection of Microorganisms, Masaryk University, Brno, Czech Republic; CCUG, Culture Collection, University of Göteborg, Göteborg, Sweden; CIP, Collection of the Institute Pasteur, Paris, France; DSM, DSMZ-Deutsche Sammlung von Mikroorganismen und Zellkulturen, Braunschweig, Germany; JCM, Japan Collection of Microorganisms, RIKEN, Saitama, Japan; LMG, LMG Culture Collection, Universiteit Gent, Gent, Belgium; NBRC, NITE Biological Resource Center, National Institute of Technology and Evaluation, Kisarazu-shi, Chiba Pref., Japan; NCCB, Netherlands Culture Collection of Bacteria, NCCB/CBS, Uppsalalaan, The Netherlands; NCIMB, National Collection of Industrial and Marine Bacteria, Aberdeen, UK. 
$\dagger$ Priority number assigned according to the date the documentation and request for validation are received.

$\ddagger$ In the article by Shukla et al. (2003), strain ATCC 700975 is erroneously cited as ATCC 700795 .

$\$$ Culture collection accession number provided on request for validation.

IIName has been corrected on validation. In previous publications, strain Aa 2.2 is cited as 'Haloferax alicantei' Holmes et al. 1997, Haloferax sp. or Haloferax phenon $\mathrm{K}$.

SCulture collection accession numbers JCM 9276, CIP 107410, DSM 14919 and CECT 5871 are also cited in the article by Gutierrez et al. (2002), but the authors did not provide certificates of deposit from these collections.

\section{References}

1. Cazemier, A. E., Verdoes, J. C., Reubsaet, F. A. G., Hackstein, J. H. P., van der Drift, C. \& Op den Camp, H. J. M. (2003). Promicromonospora pachnodae sp. nov., a member of the (hemi)cellulolytic hindgut flora of larvae of the scarab beetle Pachnoda marginata. Antonie van Leeuwenhoek 83, 135-148.

2. Gutierrez, M. C., Kamekura, M., Holmes, M. L., Dyall-Smith, M. L. \& Ventosa, A. (2002). Taxonomic characterization of Haloferax sp. (“H. alicantei") strain Aa 2.2: description of Haloferax lucentensis sp. nov. Extremophiles 6, 479-483.

3. Mori, K., Kim, H., Kakegawa, T. \& Hanada, S. (2003). A novel lineage of sulfate-reducing microorganisms: Thermodesulfobiaceae fam. nov., Thermodesulfobium narugense, gen. nov., sp. nov., a new thermophilic isolate from a hot spring. Extremophiles 7, $283-290$
4. Nakajima, Y., Ho, C. C. \& Kudo, T. (2003). Microtetraspora malaysiensis sp. nov., isolated from Malaysian primary dipterocarp forest soil. J Gen Appl Microbiol 49, 181-189.

5. Santos, P., Pinhal, I., Rainey, F. A., Empadinhas, N., Costa, J., Fields, B., Benson, R., Veríssimo, A. \& da Costa, M. S. (2003). Gamma-proteobacteria Aquicella lusitana gen. nov., sp. nov., and Aquicella siphonis sp. nov. infect protozoa and require activated charcoal for growth in laboratory media. Appl Environ Microbiol 69, 6533-6540.

6. Shukla, S. K., Bernard, K. A., Harney, M., Frank, D. N. \& Reed, K. D. (2003). Corynebacterium nigricans sp. nov. proposed name for a black-pigmented Corynebacterium species recovered from the human female urogenital tract. J Clin Microbiol 41, 4353-4358.

7. Wolin, M. J., Miller, T. L., Collins, M. D. \& Lawson, P. A. (2003). Formate-dependent growth and homoacetogenic fermentation by a bacterium from human feces: description of Bryantella formatexigens gen. nov., sp. nov. Appl Environ Microbiol 69, 6321-6326. 